\title{
AN EXTENSION WHICH IS A WEAKLY LINDELÖFF SPACE
}

\author{
YONG SIK YUN ${ }^{\mathrm{a}}$ AND ChangIL KIM ${ }^{\mathrm{b}, *}$
}

\begin{abstract}
In this paper, we construct an extension $\left(k X, k_{X}\right)$ of a space $X$ such that $k X$ is a weakly Lindelöff space and for any continuous map $f: X \longrightarrow Y$, there is a continuous map $g: k X \longrightarrow k Y$ such that $\left.g\right|_{X}=f$. Moreover, we show that $v X$ is Lindelöff if and only if $k X=v X$ and that for any $P^{\prime}$-space $X$ which is weakly Lindelöff, $k X=v X$.
\end{abstract}

\section{INTRODUCTION}

All spaces in this paper are assumed to be Tychonoff spaces and $\beta X(v X$, resp.) denotes the Stone-Čech compactification(the Hewitt realcompactification, resp.) of a space $X$.

One of the many charaterizations of $\left(\beta X, \beta_{X}\right)$ is following :

(1) $\beta X$ is a compact space, and

(2) for any continuous map $f: X \longrightarrow Y$, there is a continuous map $f^{\beta}: \beta X \longrightarrow$ $\beta Y$ such that $\left.f^{\beta}\right|_{X}=f([5])$.

There have been many ramifications from the Stone-Čech compactifications of spaces. In fact, realcompactifications of spaces and zero-dimesional compactifisations of zerodimensional spaces have been studied by various authors ([3], [5]).

The purpose to write this paper is to construct an extension of a space which has similar properties to the above extensions. We first construct an extension $\left(k X, k_{X}\right)$ of a space $X$ such that $v X \subseteq k X \subseteq \beta X$ and $k X$ is a weakly Lindelöff space. We show that for any continuous map $f: X \longrightarrow Y$, there is a continuous map $g: k X \longrightarrow k Y$ such that $\left.g\right|_{X}=f$. Blasco $([1],[2])$ showed that for a paracompact (or separable) space $X, v X$ is a Lindelöff space if and only if every separating nest generated intersection ring on $X$ is complete. We show that $v X$ is Lindelöff if and

Received by the editors April 9, 2012. Revised June 4, 2012. Accepted June 21, 2012.

2000 Mathematics Subject Classification. 54D80, 54D60, 54D20.

Key words and phrases. filter, realcompactification, weakly Lindelöff space.

${ }^{*}$ Corresponding author. 
only if $k X=v X$. Using these, we then show that $k X=X$ if and only if $X$ is Lindelöff. Finally, we will show that for any $P^{\prime}$-space $X$ which is weakly Lindelöff, $k X=v X$.

For the terminology, we refer to [3] and [5].

\section{An Extnsion Which is a Weakly Lindelöff Space}

For any space $X$, let $Z(X)$ be the set of all zero-sets in $X$. A $Z(X)$-filter is called a $z$-filter on $X$.

Definition 2.1. Let $X$ be a space and $\mathcal{F}$ a $z$-filter on $X$. Then $\mathcal{F}$ is called

(1) real if it has the countable intersection property, and

(2) free(fixed, resp.) if $\cap\{F \mid F \in \mathcal{F}\}=\emptyset(\cap\{F \mid F \in \mathcal{F}\} \neq \emptyset$, resp.).

A space $X$ is called a realcompact space if every real $z$-ultrafilter on $X$ is fixed. It is known that for any real $z$-ultrafilter $\mathcal{F}$ on a space $X, \cap\left\{c l_{v X}(F) \mid F \in \mathcal{F}\right\} \neq \emptyset([3])$.

Let $X$ be a space and $k X=v X \cup\{p \in \beta X-v X \mid$ there is a real $z$-filter $\mathcal{F}$ on $X$ such that $\cap\left\{c l_{v X}(F) \mid F \in \mathcal{F}\right\}=\emptyset$ and $\left.p \in \cap\left\{c l_{\beta X}(F) \mid F \in \mathcal{F}\right\}\right\}$.

Let $X$ be a set and $\mathcal{F} \subseteq \mathcal{P}(X)$. For any $A \subseteq X$, let $\mathcal{F}_{A}$ denote the set $\{F \cap A \mid$ $F \in \mathcal{F}\}$.

Proposition 2.2. Let $X$ be a space. Then we have the following:

(1) $v X \subseteq k X \subseteq \beta X$,

(2) $k(v X)=k X$, and

(3) $k X$ is realcompact if for any non-empty zero-set $Z$ in $k X, Z \cap X \neq \emptyset$.

Proof. (1) It is trivial.

(2) Let $p \in k X-v X$. Then there is a real $z$-filter $\mathcal{F}$ on $X$ such that $\cap\left\{c l_{v X}(F) \mid\right.$ $F \in \mathcal{F}\}=\emptyset$ and $p \in \cap\left\{c l_{\beta X}(F) \mid F \in \mathcal{F}\right\}$. Let $\mathcal{F}_{v}=\left\{c l_{v X}(F) \mid F \in \mathcal{F}\right\}$. Note that for any zero-set $Z$ in $X, c_{v X}(Z)$ is a zero-set in $v X$ and for any sequence $\left(Z_{n}\right)$ in $Z(X), c_{v X}\left(\cap\left\{Z_{n} \mid n \in N\right\}\right)=\cap\left\{c l_{v X}\left(Z_{n}\right) \mid n \in N\right\}([3])$. Hence $\mathcal{F}_{v}$ is a real $z$-filter $\mathcal{F}$ on $v X$. Note that $\cap\left\{c_{v X}(H) \mid H \in \mathcal{F}_{v}\right\}=\left\{c l_{v X}(F) \mid F \in \mathcal{F}\right\}=\emptyset$ and $p \in \cap\left\{c l_{\beta X}(H) \mid H \in \mathcal{F}_{v}\right\}=\cap\left\{c l_{\beta X}(F) \mid F \in \mathcal{F}\right\}$. Since $v(v X)=v X$ and $\beta(v X)=\beta X, p \in k(v X)$. Hence $k X \subseteq k(v X)$.

Let $q \in k(v X)$ and $q \notin v X$. Since $v(v X)=v X$, there is a real $z$-filter $\mathcal{G}$ on $v X$ such that $\cap\{G \mid G \in \mathcal{G}\}=\emptyset$ and $q \in \cap\left\{c l_{\beta X}(G) \mid G \in \mathcal{G}\right\}$. Then $\mathcal{G}_{X}$ is a real $z$-filter on $X$ and $\cap\left\{c_{v X}(H) \mid H \in \mathcal{G}_{X}\right\}=\cap\{G \mid G \in \mathcal{G}\}=\emptyset$. Since $q \in \cap\left\{c l_{\beta X}(H) \mid H \in \mathcal{G}_{X}\right\}=\cap\left\{c l_{\beta X}(G) \mid G \in \mathcal{G}\right\}, q \in k X$. Hence $k(v X) \subseteq k X$.

(3) Take any real $z$-ultrafilter $\mathcal{F}$ on $k X$. By the assumption, for any $F \in \mathcal{F}$, 
$F \cap X \neq \emptyset$ and so $\mathcal{F}_{X}$ is a $z$-filter on $X$. Let $Z$ be a zero-set in $X$ such that for any $F \in \mathcal{F}, Z \cap F \neq \emptyset$. Since $X \subseteq k X \subseteq \beta X$, there is a zero-set $B$ in $k X$ such that $Z=B \cap X$. Then for any $F \in \mathcal{F}, F \cap B \neq \emptyset$. Since $\mathcal{F}$ is a $z$ ultrafilter on $k X, B \in \mathcal{F}$ and $B \cap X=Z \in \mathcal{F}_{X}$. Hence $\mathcal{F}_{X}$ is a $z$-ultrafilter on $X$. Since $\mathcal{F}_{X}$ is real, $\cap\left\{c l_{v X}(F \cap X) \mid F \in \mathcal{F}\right\}=\{q\}$ for some $q \in v X$. Note that $\cap\left\{c l_{v X}(F \cap X) \mid F \in \mathcal{F}\right\}=\cap\left\{c_{v X}(F \cap v X) \mid F \in \mathcal{F}\right\}$ and for any $F \in \mathcal{F}$, $c l_{v X}(F \cap v X) \subseteq F$. Hence $q \in \cap\{F \mid F \in \mathcal{F}\}$ and so $\cap\{F \mid F \in \mathcal{F}\} \neq \emptyset$. Thus $k X$ is a realcompact space.

Let $S$ be a subspace of a space $X$. Then $S$ is called $C\left(C^{*}\right.$, resp. $)$-embedded in $X$ if for any real-valued (bounded, resp.) continuous function $f$ on $S$, there is a real-valued (bounded, resp.) continuous function $g$ on $X$ such that $\left.g\right|_{S}=f$.

Note that $X$ is a dense $C$-embedded subspace of $Y$ if and only if $X \subseteq Y \subseteq v X$, equivalently, $v X=v Y$ and that a dense subspace $X$ of a space $Y$ is $C^{*}$-embedded in $Y$ if and only if $\beta X=\beta Y$ ([3]). Using these, we have the following :

Proposition 2.3. Let $X$ be a dense $C$-embedded subspace of $Y$. Then $k X=k Y$.

Proof. Since $X$ is a dense $C$-embedded subspace of $Y, v X=v Y([3])$. Let $p \in$ $k X-v X$. Then there is a real $z$-filter $\mathcal{F}$ on $X$ such that $\cap\left\{c l_{v X}(F) \mid F \in \mathcal{F}\right\}=\emptyset$ and $p \in \cap\left\{c l_{\beta X}(F) \mid F \in \mathcal{F}\right\}$. Let $\mathcal{G}=\{G \in Z(Y) \mid G \cap X \in \mathcal{F}\}$. Then $\mathcal{G}_{X}=\mathcal{F}$ and since $v X=v Y, \mathcal{G}$ is a real $z$-filter on $Y$.

Let $G \in \mathcal{G}$ and $x \in v X-c l_{v X}(G \cap X)$. Then there is a zero-set neighborhood $Z$ of $x$ in $v X$ such that $G \cap X \cap Z=\emptyset$. Since $X \subseteq Y \subseteq v X$, there is a zero-set $H$ in $v X$ such that $G=H \cap Y$. Since $H \cap Z \cap X=\emptyset$ and $H \cap Z$ is a zero-set in $v X$, $H \cap Z=\emptyset([5])$. Hence $G \cap Z=\emptyset$ and $x \notin c l_{v X}(G)$. Thus $c_{v X}(G) \subseteq c_{v X}(G \cap X)$. Clearly, $c_{v X}(G \cap X) \subseteq \operatorname{cl}_{v X}(G)$ and so $c_{v X}(G \cap X)=c l_{v X}(G)$.

Since $\cap\left\{c_{v X}(G \cap X) \mid G \in \mathcal{G}\right\}=\emptyset, \cap\left\{c l_{v Y}(G) \mid G \in \mathcal{G}\right\}=\emptyset$. Since $X$ is $C^{*}$ embedded in $Y, \beta X=\beta Y$ and $p \in \cap\left\{c l_{\beta Y}(G) \mid G \in \mathcal{G}\right\}$. Hence $p \in k Y$ and so $k X \subseteq k Y$.

Similarly, we have $k Y \subseteq k X$.

For any space $X$, let $k_{X}: X \longrightarrow k X$ denote the inclusion map. Then $\left(k X, k_{X}\right)$ is an extension of $X$.

Note that for any continuous map $f: X \longrightarrow Y$, there is a unique continuous map $f^{v}: v X \longrightarrow v Y$ such that $\left.f^{v}\right|_{X}=f$.

Proposition 2.4. Let $f: X \longrightarrow Y$ be a continuous map. Then there is a unique continuous map $g: k X \longrightarrow k Y$ such that $g \circ k_{X}=k_{Y} \circ f$. 
Proof. Note that there is a continuous map $h: \beta X \longrightarrow \beta Y$ such that $h \circ \beta_{X}=\beta_{Y} \circ f$ and $h(v X) \subseteq v Y$. Let $p \in k X-v X$. Then there is a real $z$-filter $\mathcal{F}$ on $X$ such that $\cap\left\{c l_{v X}(F) \mid F \in \mathcal{F}\right\}=\emptyset$ and $p \in \cap\left\{c l_{\beta X}(F) \mid F \in \mathcal{F}\right\}$. Let $\mathcal{G}=\{Z \in Z(Y) \mid$ $\left.h^{-1}(Z) \in \mathcal{F}\right\}$. Since $\mathcal{F}$ is a real $z$-filter on $X, \mathcal{G}$ is a real $z$-filter on $Y$. Let $G \in \mathcal{G}$. Then $h^{-1}(G) \in \mathcal{F}$. Since $p \in c l_{\beta X}\left(h^{-1}(G)\right), h(p) \in h\left(c_{\beta X}\left(h^{-1}(G)\right)\right) \subseteq$ $c l_{\beta Y}\left(h\left(h^{-1}(G)\right)\right) \subseteq c_{\beta Y}(G)$. Hence $h(p) \in \cap\left\{c l_{\beta X}(G) \mid G \in \mathcal{G}\right\}$ and so $h(p) \in k Y$. Let $g: k X \longrightarrow k Y$ be the restriction and corestriction of $h$ with respect to $k X$ and $k Y$, respectively. Then $g: k X \longrightarrow k Y$ is a continuous map and $g \circ k_{X}=k_{Y} \circ f$. Since $k_{X}: X \longrightarrow k X$ is a dense embedding, such an $g$ is unique.

It is well-known that a space $X$ is Lindelöff if and only if for any real $z$-filter $\mathcal{F}$ in $X, \cap\{F \mid F \in \mathcal{F}\} \neq \emptyset$.

Proposition 2.5. Let $X$ be a space. Then the following are equivalent :

(1) $v X=k X$,

(2) $v X$ is a Lindelöff space,

(3) for any free real $z$-filter $\mathcal{F}$ on $X, \cap\left\{\operatorname{cl}_{v X}(F) \mid F \in \mathcal{F}\right\} \neq \emptyset$, and

(4) for any free real $z$-filter $\mathcal{F}$ on $X$, there is a free real $z$-ultrafilter $\mathcal{A}$ on $X$ such that $\mathcal{F} \subseteq \mathcal{A}$.

Proof. (1) $\Rightarrow(2)$ Take any real $z$-filter $\mathcal{G}$ on $v X$. Then $\mathcal{G}_{X}$ is a real $z$-filter on $X$. Suppose that $\cap\{G \cap X \mid G \in \mathcal{G}\}=\emptyset$. Then $\cap\left\{c l_{\beta X}(G \cap X) \mid G \in \mathcal{G}\right\} \neq \emptyset$. Pick $p \in \cap\left\{c l_{\beta X}(G \cap X) \mid G \in \mathcal{G}\right\}$. Then $p \in k X$ and since $k X=v X, p \in v X$. Hence $p \in\left(\cap\left\{c_{\beta X}(G) \mid G \in \mathcal{G}\right\}\right) \cap v X=\cap\{G \mid G \in \mathcal{G}\}$ and so $\cap\{G \mid G \in \mathcal{G}\} \neq \emptyset$. Thus $v X$ is a Lindelöff space.

$(2) \Rightarrow(3)$ It is trivial.

$(3) \Rightarrow(4)$ Let $\mathcal{F}$ be a free real $z$-filter on $X$. By the assumption, $\cap\left\{c l_{v X}(F) \mid\right.$ $F \in \mathcal{F}\} \neq \emptyset$. Pick $p \in \cap\left\{c l_{v X}(F) \mid F \in \mathcal{F}\right\}$. Let $\mathcal{A}^{p}=\left\{A \in Z(X) \mid p \in c l_{v X}(A)\right\}$. Then $\mathcal{A}^{p}$ is a free real $z$-ultrafilter on $X$ and $\mathcal{F} \subseteq \mathcal{A}^{p}$.

(4) $\Rightarrow(1)$ Let $p \in k X-v X$. Then there is a real $z$-filter $\mathcal{F}$ on $X$ such that $\cap\left\{c_{v X}(F) \mid F \in \mathcal{F}\right\}=\emptyset$ and $p \in \cap\left\{c_{\beta X}(G \cap X) \mid G \in \mathcal{G}\right\}$. Since $\mathcal{F}$ is free, by (4), there is a free real $z$-ultrafilter $\mathcal{A}$ on $X$ such that $\mathcal{F} \subseteq \mathcal{A}$. Since $\cap\left\{c l_{v X}(A) \mid A \in\right.$ $\mathcal{A}\} \neq \emptyset, \cap\left\{c l_{v X}(F) \mid F \in \mathcal{F}\right\} \neq \emptyset$ and this is a contradiction.

By Proposition 2.2. and Proposition 2.5., we have the following :

Corollary 2.6. Let $X$ be a sapce. Then $k X=X$ if and only if $X$ is Lindelöff.

Recall that a space $X$ is called a pseudo-compact space if every real-valued 
continuous function on $X$ is bounded, equivalently, $v X=\beta X$.

Corollary 2.7. If $X$ is a pseudo-compact space, then $k X=\beta X$.

Let $X$ be a space. The collection $\mathcal{R}(X)$ of all regular closed sets in $X$, when partially ordered by inclusion, becomes a complete Boolean algebra, in which the join, meet, and complementation operations are defined as follows : For any $A \in$ $\mathcal{R}(X)$ and any $\mathcal{F} \subseteq \mathcal{R}(X)$

$$
\begin{aligned}
& \bigvee \mathcal{F}=c l_{X}(\cup\{F \mid F \in \mathcal{F}\}), \\
& \bigwedge \mathcal{F}=c l_{X}\left(i n t_{X}(\cap\{F \mid F \in \mathcal{F}\})\right), \text { and } \\
& A^{\prime}=c l_{X}(X-A) .
\end{aligned}
$$

A sublattice of $\mathcal{R}(X)$ is a subset of $\mathcal{R}(X)$ that contains $\emptyset, X$ and is closed under finite joins and finite meets $([7])$.

An $\mathcal{R}(X)$-filter $\mathcal{A}$ is said to have the countable meet property if for any sequence $\left(A_{n}\right)$ in $\mathcal{R}(X), \bigwedge\left\{A_{n} \mid n \in N\right\} \neq \emptyset$.

Let $Z(X)^{\#}=\left\{c l_{X}\left(\operatorname{int}_{X}(A)\right) \mid A \in Z(X)\right\}$. Then $Z(X)^{\#}$ is a sublattice of $R(X)$.

A space $X$ is called a weakly Lindelöff space if for any open cover $\mathcal{U}$ of $X$, there is a countable subset $\mathcal{V}$ of $\mathcal{U}$ such that $\cup\{V \mid V \in \mathcal{V}\}$ is dense in $X$.

A space $X$ is a weakly Lindelöff space if and only if for any $Z(X)^{\#}$-filter $\mathcal{A}$ with the countable meet property, $\cap\{A \mid A \in \mathcal{A}\} \neq \emptyset([4])$.

Theorem 2.8. Let $X$ be a space. Then $k X$ is a weakly Lindelöff space.

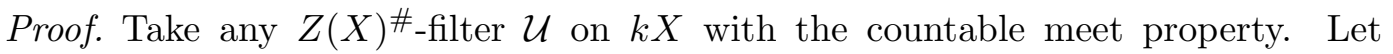
$\mathcal{F}=\left\{Z \in Z(k X) \mid c_{k X}\left(\right.\right.$ int $\left.\left._{k X}(Z)\right) \in \mathcal{U}\right\}$. Clearly, $\emptyset \notin \mathcal{F} \neq \emptyset$. For any $A, B \in \mathcal{F}$, $c l_{k X}\left(\right.$ int $\left._{k X}(A \cap B)\right)=c l_{k X}\left(\right.$ int $\left._{k X}(A)\right) \wedge c l_{k X}\left(\right.$ int $\left._{k X}(B)\right) \in \mathcal{U}$ and hence $A \cap B \in \mathcal{F}$. Thus $\mathcal{F}$ is a $z$-filter on $k X$. By the definition of $\mathcal{F}$, for any $F \in \mathcal{F}, F \cap X \neq \emptyset$. Hence $\mathcal{F}_{X}$ is also a $z$-filter on $X$. Let $\left(A_{n}\right)$ be a sequence in $\mathcal{F}_{X}$. For any $n \in N$, there is a $B_{n} \in \mathcal{F}$ such that $A_{n}=B_{n} \cap X$. Since $\mathcal{U}$ has the countable meet property, $c_{k X}\left(\operatorname{int}_{k X}\left(\cap\left\{B_{n} \mid n \in N\right\}\right)\right) \neq \emptyset$ and since $X$ is dense in $k X, \operatorname{cl}_{k X}\left(\operatorname{int}_{k X}\left(\cap\left\{B_{n} \mid\right.\right.\right.$ $n \in N\})) \cap X \neq \emptyset$. Since $c l_{k X}\left(\operatorname{int}_{k X}\left(\cap\left\{B_{n} \mid n \in N\right\}\right)\right) \cap X$

$$
\begin{aligned}
& =c_{k X}\left(\operatorname{int}_{k X}\left(\cap\left\{B_{n} \cap X \mid n \in N\right\}\right)\right) \\
& =c_{k X}\left(\operatorname{int}_{k X}\left(\cap\left\{A_{n} \mid n \in N\right\}\right)\right),
\end{aligned}
$$

$\cap\left\{A_{n} \mid n \in N\right\} \neq \emptyset$ and so $\mathcal{F}_{X}$ has the countable intersection property. Note that $\cap\left\{c l_{v X}(F \cap X) \mid F \in \mathcal{F}\right\} \neq \emptyset$ or $\cap\left\{c l_{v X}(F \cap X) \mid F \in \mathcal{F}\right\}=\emptyset$.

Assume that $\cap\left\{\operatorname{cl}_{v X}(F \cap X) \mid F \in \mathcal{F}\right\} \neq \emptyset$. Pick $x \in \cap\left\{c_{v X}(F \cap X) \mid F \in \mathcal{F}\right\}$. Let $U \in \mathcal{U}$. Suppose that $x \notin U$. Since $U$ is a closed set in $k X$, there is a zero-set $Z$ in $k X$ such that $x \notin Z$ and $U \subseteq Z$. Then $Z \cap X \in \mathcal{F}_{X}$ and since $c l_{v X}(Z \cap X)=Z \cap v X$, 
since $c_{v X}(Z \cap X)=Z \cap v X, x \in Z$. This is a contradiction and so $x \in U$. Hence $x \in \cap\{U \mid U \in \mathcal{U}\}$.

Assume that $\cap\left\{\operatorname{cl}_{v X}(F \cap X) \mid F \in \mathcal{F}\right\}=\emptyset$. Let $p \in \cap\left\{c_{\beta X}(F \cap X) \mid F \in \mathcal{F}\right\}$. Then $p \in k X$. Let $U \in \mathcal{U}$. Suppose that $p \notin U$. Then there is a zero-set $B$ in $\beta X$ such that $p \notin B$ and $U \subseteq B$. Since $B \cap X \in \mathcal{F}_{X}, p \in c l_{\beta X}(B \cap X) \subseteq B$. This is a contradiction and so $p \in U$. Hence $p \in \cap\{U \mid U \in \mathcal{U}\}$.

Thus $\cap\{U \mid U \in \mathcal{U}\} \neq \emptyset$ and $k X$ is a weakly Lindelöff space.

A space $X$ is called a $P^{\prime}$-space if for any non-empty zero-set $Z$ in $X, \operatorname{int}_{X}(Z) \neq \emptyset$, equivalently, every zero-set in $X$ is a reqular closed set in $X$. Clearly, a space $X$ is a $P^{\prime}$-space if and only if $v X$ is a $P^{\prime}$-space. If $X$ is a realcompact and locally compact space, then $\beta X-X$ is a $P^{\prime}$-space $([6])$.

Proposition 2.9. Let $X$ be a $P^{\prime}$-space. Then $X$ is a weakly Lindelöff space if and only if $X$ is a Lindelöff space.

Proof. Suppose that $X$ is a weakly Lindelöff space. Let $\mathcal{F}$ be a real $z$-filter on $X$. Since $X$ is a $P^{\prime}$-space, $Z(X)=Z(X)^{\#}$ and since $Z(X)$ is closed under countable intersections, for any sequence $\left(A_{n}\right)$ in $Z(X)$,

$$
\bigwedge\left\{A_{n} \mid n \in N\right\}=\operatorname{cl}_{X}\left(\operatorname{int}_{X}\left(\cap\left\{A_{n} \mid n \in N\right\}\right)\right)=\cap\left\{A_{n} \mid n \in N\right\} .
$$

Hence $\mathcal{F}$ is a $Z(X)^{\#}$-filter with the countable meet property. Since $X$ is a weakly Lindelöff space, $\cap\{F \mid F \in \mathcal{F}\} \neq \emptyset$ and hence $X$ is a Lindelöff space.

The converse is trivial.

A space with a dense weakly Lindelöff space is also a weakly Lindelöff space. Using this, Proposition 2.9. and Proposition 2.5., we have the following :

Corollary 2.10. For any $P^{\prime}$-space $X$ which is weakly Lindelöff, $v X$ is a Lindelöff space and $v X=k X$.

\section{REFERENCES}

1. J.L. Blasco: Complete bases and Wallman realcompactifications. Proc. Amer. Math. Soc. 75 (1979), 114-118.

2. ___ Complete bases in topological spaces II. Studia Sci. Math. Hung. 24 (1989), 447-452.

3. L. Gillman \& M. Jerison: Rings of continuous functions. Van Nostrand, Princeton, New York, (1960).

4. C.I. Kim: Almost P-spaces. Commun. Korean Math. Soc. 18 (2003), 695-701. 
5. J. Porter \& R.G. Woods: Extensions and Absolutes of Hausdorff Spaces. Springer, Berlin, (1988).

6. A.I. Veksler: $P^{\prime}$-points, $P^{\prime}$-sets, $P^{\prime}$-spaces. A new class of order-continuous measures and functionals, Soviet Math. Dokl. 14 (1973), 1445-1450.

${ }^{a}$ Department of Mathematics, Jeju National University, Jeju 690-756, Korea

Email address: yunys@jejunu.ac.kr

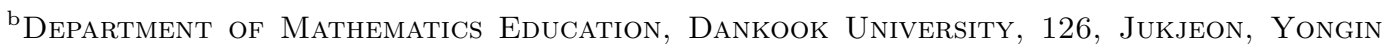
448-701, KOREA

Email address: kci206@hanmail.net 